\title{
SUPPLEMENTARY MATERIAL FOR "THE POTENTIAL FOR BIAS IN PRINCIPAL CAUSAL EFFECT ESTIMATION WHEN TREATMENT RECEIVED DEPENDS ON A KEY COVARIATE"
}

\author{
BY CORWIN M. ZIGLER ${ }^{* \dagger}$ AND THOMAS R. BELIN ${ }^{\ddagger}$ \\ Harvard University ${ }^{\dagger}$ and University of California, Los Angeles
}

\begin{abstract}
SIMULATION STUDY
We simulate several data scenarios inspired by the motivating oral-surgery example, varying the relationships between injury severity and stratum membership and between injury severity and the clinical outcome. The data are simulated as follows: 1) datasets of $n=300$ are simulated consisting of equal proportions of never-takers, compliers, and always-takers; 2) $S E V$ is simulated from a normal distribution in each stratum with a common variance across strata and the extent of the mean differences varied in the different datasets; 3) GOHAI values are simulated from normal distributions with means dependent on stratum membership and treatment receipt, variances constant across all groups, and correlations with injury severity that are varied in the different datasets; 4) observations are randomly assigned in equal proportion to the two treatment groups, and unobservable potential outcomes are set to missing. For each simulated dataset, we set the mean $S E V$ in the never-takers, compliers, and always-takers to be $12.5,12.5+\delta_{X}, 12.5+2 \delta_{X}$, respectively, where $\delta_{X}=0.0,0.25,0.5,1.0$, or 2.0 standard deviations. For each value of $\delta_{X}$, we simulate datasets with correlation between $S E V$ and GOHAI set to $\rho=0.0,-0.1,-0.2,-0.3,-0.4$, or -0.5 in each stratum to represent injury severity having no effect or an adverse effect on oral health. To simulate scenarios with a CACE $=0$ and with realistic GOHAI values, we set mean GOHAI values to 42 and 48 in the always-takers and never-takers, respectively, and complier mean GOHAI values to 45 in both treatment groups, with a standard deviation of 10 in all groups. Injury severity was simulated to have a standard deviation of 2.5. 50 datasets are simulated and analyzed under each scenario to ensure that illustrative results are not obscured by sampling variability.
\end{abstract}

A.1. Impact of the compliance-predictive model on the estimation of principal stratum membership. To illustrate that the compliance-predictive model can imply complier treatment groups with different characteristics, we contrast in detail the analyses of two simulated datasets representing scenarios where 1) $X$ does not predict stratum membership $\left(\delta_{X}=0\right)$ and 2) $X$ predicts stratum membership $\left(\delta_{X}=1\right)$. Both of these datasets have $\rho=0$ to represent scenarios where $X$ does not relate to $Y$.

We first perform the analysis under Model A, which correctly models the data as simulated. To investigate the relationships between $S E V$ and stratum membership, we examine posterior predictive distributions of the quantities in (4.8). To this end, we consider a hypothetical "new" set of patients with $X$ across the entire range observed in the sample, $Y$ set to the observed sample mean, and $Z_{i}=D_{i}^{o b s}=z$ for $z=0,1$. Figures 1(a) and 1(b) depict for each value of $z$ the posterior predictive means and $95 \%$ intervals of the probability of being sampled as a complier (4.8) across the range of $X$. For the scenario with $\delta_{X}=0$, we see in Figure 1(a) that the Bernoulli probabilities of membership in the compliance stratum are similar across the range of $X$ in both patients with $Z_{i}=D_{i}^{o b s}=0$ and those with $Z_{i}=D_{i}^{o b s}=1$, reflecting the inability of the covariate to distinguish between strata.

However, when $X$ is predictive of stratum membership as in the scenario with $\delta_{X}=1$, the analogous Figure 1(b) displays that the relationships between $X$ and the probability of being sampled as a complier differ depending on the value of $Z_{i}$ and $D_{i}^{o b s}$. Specifically, we see that a patient with $Z_{i}=D_{i}^{o b s}=0$ and low $X$ will have a low probability of being sampled as a complier, while a patient with identical $X$ who has

\footnotetext{
${ }^{*}$ Corresponding author
} 
$Z_{i}=D_{i}^{o b s}=1$ will have a comparatively high probability of being sampled as a complier (with the opposite relationship at the high end of the $X$ distribution).

To determine whether the procedure implies compliers who differ with respect to $X$, we examine posteriorpredictive distributions of the probabilities in (4.8) for two new sets of hypothetical patients having $X$ distributions identical to those observed in the data for patients with $Z_{i}=D_{i}^{o b s}=z$, for $z=0,1$. In the simulated dataset with $\delta_{X}=0$, Figure 2(a) displays, for $z=0,1$, histograms of the observed $X$ distributions in patients with $Z_{i}=D_{i}^{o b s}=z$, with histogram bars shaded according to the mean posterior-predictive probability of membership in the complier stratum for a value of $X$ at that point of the histogram and for $Y_{i}^{o b s}$ equal to the mean value observed in patients with $Z_{i}=D_{i}^{o b s}=z$. Figure 2(a) provides no indication of differential sampling of compliers, as the similar shading pattern across the $X$ histograms in both treatment groups implies that compliers are selected from the same portion of the $X$ distribution in both arms.

Figure 2(b), generated analogously but for the simulated dataset with $\delta_{X}=1$, indicates a different relationship where the procedure tends to draw treatment and control compliers from opposite ends of the $X$ distribution. For example, note that patients with $X$ in the range $[9,12]$ in the $Z_{i}=D_{i}^{o b s}=1$ group have probability of membership in the complier stratum near 1.0, while patients with the same range of $S E V$ in the $Z_{i}=D_{i}^{o b s}=0$ group have probability of membership in the complier stratum in the range $[0.1-0.4]$. The implication for estimates of the CACE is that over the course of the sampler, patients in the observed mixture of compliers and always-takers $\left(Z_{i}=D_{i}^{o b s}=1\right)$ with lower $X$ will more often contribute to the CACE than patients with comparable $X$ in the observed mixture of compliers and never-takers $\left(Z_{i}=D_{i}^{o b s}=0\right)$. The opposite sampling disparity holds for patients with higher $X$. Thus, Figure 2(b) indicates the extent to which our model leads to estimates of the CACE that rely on areas of the $X$ distribution where the availability of compliers from both treatment arms is limited.

A situation such as that depicted in Figure 2(b) leaves estimates of the CACE particularly vulnerable to impacts of misspecified models that fail to accurately capture the true relationships between $X$ and $Y$ or extrapolate to areas of the $X$ distribution where there is limited data. To illustrate this point, we repeat the analyses described above under Model B, which estimates the CACE without adjustment for $X$. Model B entails a typical unadjusted CACE estimate that might be employed in many settings, but in this simulated example it also represents a misspecified model.

Under the misspecified Model B, figures analogous to Figures 1 and 2 appear very similar to those produced under Model A and are not pictured. This is because the $\Psi\left(X_{i}, \beta\right)$ terms dominate the probability in (4.8) and the slight alteration of the other terms in this expression leaves these probabilities virtually unchanged. However, Model B can yield vastly different estimates of the CACE.

Figure 3 displays the performance of the procedure for estimating the CACE under both Model A (red line) and Model B (black line) using the average posterior mean and 95\% posterior intervals calculated across the 50 replicated datasets for each of the simulated scenarios. Under both models, the precision of the estimates increases with the value of $\delta_{X}$; the better $X$ predicts stratum membership, the larger the gain in precision. However, we see that under Model B, the imbalanced sampling of compliers evident from a figure such as Figure 2 leads to bias in the estimated CACE that is increasing in $|\rho|$ when $\delta_{X}>0$, providing misleading results even when the association between $X$ and $Y$ is modest and in some cases estimating a significant treatment effect when there in fact is none. The same bias is not depicted under Model A because even though there is limited data on comparable compliers at the tails of the $X$ distribution, extrapolation of Model A to these areas of the distribution correctly reflects the underlying relationship.

CORWIN M. ZIGLER

HaRVARd SCHOOL OF Public Health

BUILDING 2, 4TH FLOOR

655 Huntington Ave

BosTON, MA 02115

E-MAIL: czigler@hsph.harvard.edu
THOMAS R. BELIN

DEPARTMENT OF BIOSTATISTICS

UCLA School of Public Health

51-267 CENTER For Health SCIENCES

LOS ANGELES, CA 90095-1772

E-MAIL: tbelin@mednet.ucla.edu 


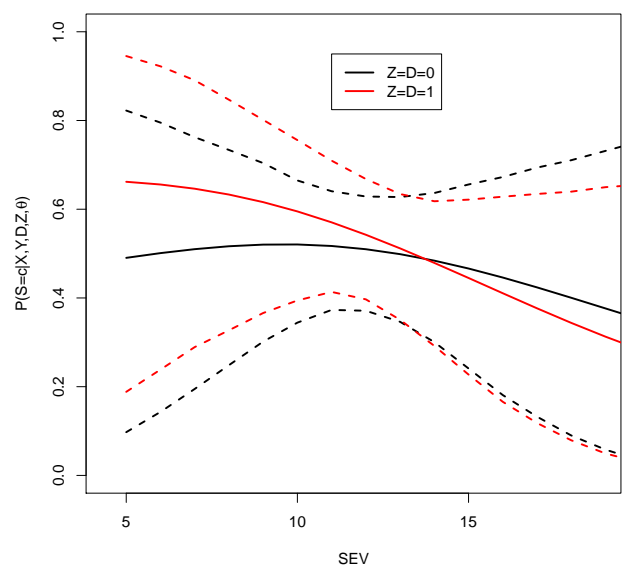

(a) $\delta_{X}=0, \rho=0$

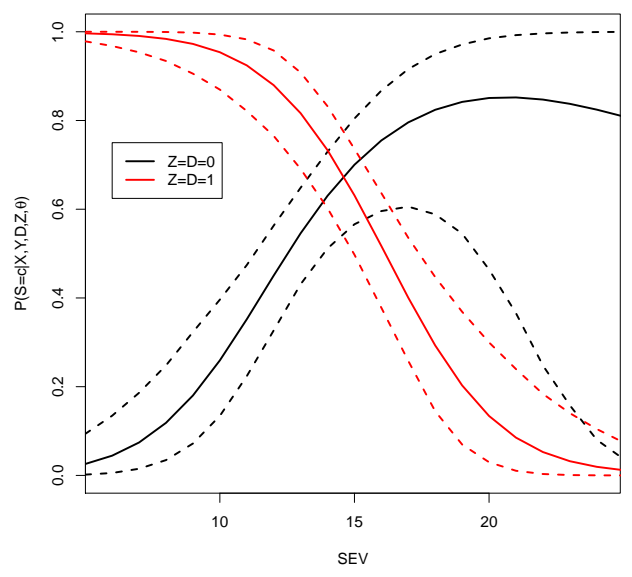

(b) $\delta_{X}=1, \rho=0$

FIG 1. Mean posterior-predictive probabilities (with $95 \%$ intervals) of $P\left(S_{i}=c \mid X_{i}, Y_{i}^{\text {obs }}, D_{i}^{\text {obs }}, Z_{i}, \theta\right)$ under Model A for hypothetical patients with $Z_{i}=D_{i}^{o b s}=z$ for $z=0,1$ and $S E V$ across the observed range in each of two simulated datasets where $S E V$ is not (a) or is (b) predictive of stratum membership.

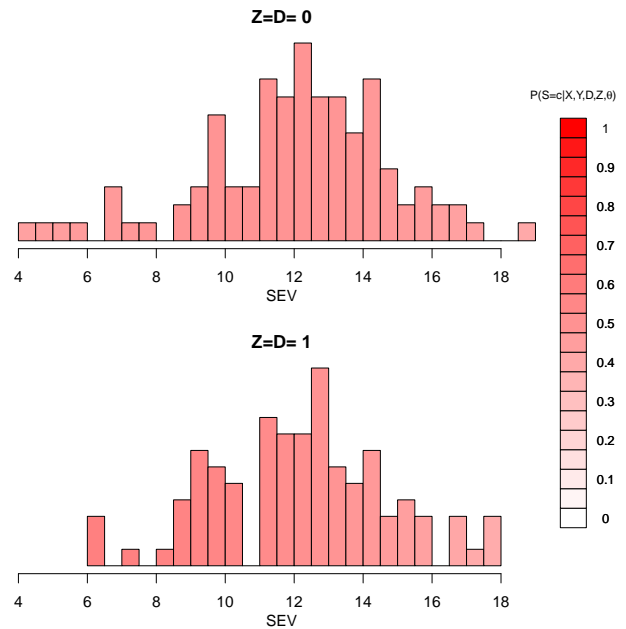

(a) $\delta_{X}=0, \rho=0$.

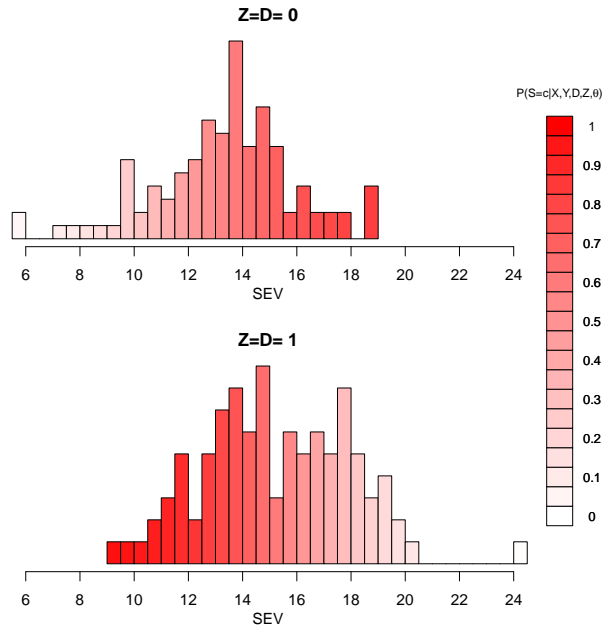

(b) $\delta_{X}=1, \rho=0$.

FIG 2. Observed SEV distributions in patients with $Z_{i}=D^{\text {obs }}=z$ for $z=0,1$ for each of two simulated datasets where $S E V$ is not (a) or is (b) predictive of stratum membership. Histogram bars are shaded corresponding to the mean posterior-predictive $P\left(S_{i}=c \mid X_{i}, Y_{i}^{o b s}, D_{i}^{o b s}, Z_{i}, \theta\right)$. 


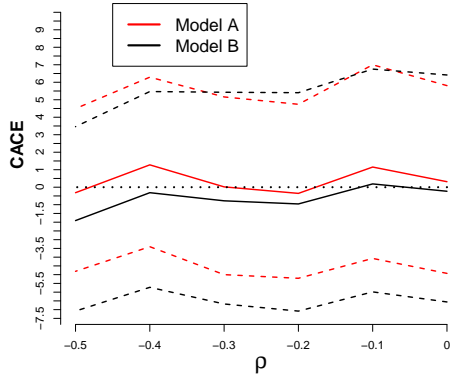

(a) $\delta_{X}=0$

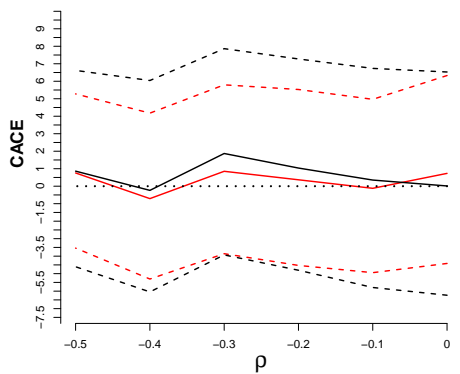

(b) $\delta_{X}=0.25$

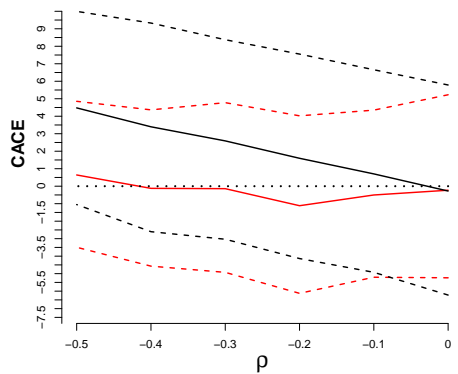

(c) $\delta_{X}=0.5$

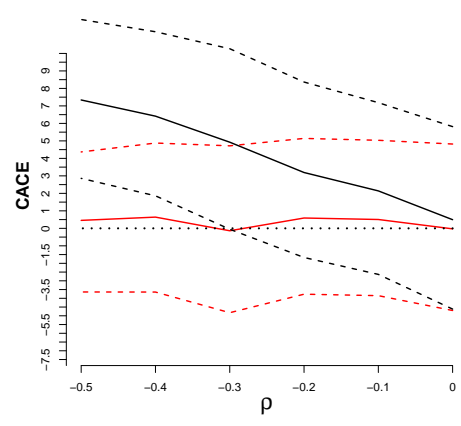

(d) $\delta_{X}=1.0$

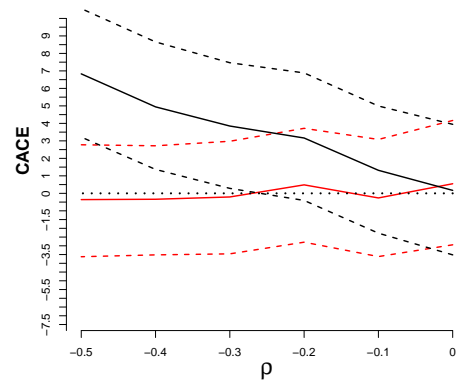

(e) $\delta_{X}=2.0$

FIG 3. Average posterior mean CACE (and 95\% intervals) across simulated datasets with different values of $\rho$ for different values of $\delta_{X}$. Posterior means and intervals are averaged across 50 replicated datasets for each value of $\rho$ and $\delta_{X}$. Horizontal dotted line is at $C A C E=0$. 